Pacific Journal of Mathematics

SUBDIFFERENTIALS OF CONVEX FUNCTIONS ON BANACH 


\title{
SUBDIFFERENTIALS OF CONVEX FUNCTIONS ON BANACH SPACES
}

\author{
GEORGE LUNA
}

This note was motivated by a paper of P. D. Taylor, which contains a simple proof of Rockafellar's basic theorem that the subdifferential map $\partial f$ of a lower semicontinuous proper convex function $f$ on a Banach space is maximal monotone. Taylor based his proof on a theorem which can be considered as a sharpening (for the epigraph of a convex function) of a result (Lemma 1.1) concerning support points and functions of convex sets due to Brondsted-Rockafellar and Phelps. It is shown that Taylor's theorem can be generalized somewhat, using related methods. (It is shown, by an example, that there is a limitation on the extent of generalization possible.) The theorem follows from a slightly technical result (Proposition 1.3) which admits a dual version (Proposition 2.2). As an application of Proposition 2.2, a short proof of Rockafellar's theorem relating the graph of $\left(\partial f^{*}\right)^{-1}$ to that of $\partial f$ is given. The methods of this paper yield a generalization (Corollary 1.9) of one of the density results of Bishop-Phelps.

1. Let $E$ be a topological vector space and $E^{*}$ its dual. The natural pairing between these spaces will be denoted by $\left\langle x, x^{*}\right\rangle$ for $x \in E$ and $x^{*} \in E^{*}$.

We recall some standard definition and facts about convex sets and functions. For more detail see Moreau [6] or Rockafellar [9]. If $f: E \rightarrow[\infty, \infty]$ is a function, then its epigraph (or "supergraph") epi $f$ is $\{(x, r) \in E \times R \mid f(x) \leqq r\}$. Recall $f$ is convex if and only if epi $f$ is convex and that $f$ is lower semicontinuous (l.s.c.) if and only if epi $f$ is closed in $E \times R$. If epi $f$ is nonempty and contains no vertical lines, i.e., sets of the form $\{(x, r) \mid r \in R\}$ where $x \in E$, then $f$ is called proper. The natural projection of epi $f$ onto $E$ is called the effective domain of $f$ and is written $\operatorname{dom} f$; thus $\operatorname{dom} f=\{x \in E \mid f(x)<\infty\}$.

If $g$ is a function on a set $X$ we write sup $g(X)$ in place of $\sup \{g(x) \mid x \in X\}$. If $C$ is a closed convex nonempty subset of $E$, a support point for $C$ is a point $x \in C$ for which there exists an element $x^{*} \in E^{*} \backslash\{0\}$ such that $\left\langle x, x^{*}\right\rangle=\sup x^{*}(C)$. Such an element $x^{*}$ is called a support functional for $C$.

We identify $(E \times R)^{*}$ and $E^{*} \times R$ in the obvious way, so that the pairing between $E^{*} \times R$ and $E \times R$ is given by $\left\langle(x, r),\left(x^{*}, s\right)\right\rangle=\left\langle x, x^{*}\right\rangle+r s$ for $(x, r) \in E \times R$ and $\left(x^{*}, s\right) \in E^{*} \times R$.

If $f$ is a convex function on $E$, a subgradient for $f$ at a point $x \in \operatorname{dom} f$ is an element $x^{*} \in E^{*}$ such that $\left(x^{*},-1\right)$ is a support func- 
tional of epi $f$ at $(x, f(x))$. The collection (possibly empty) of all subgradients at $x$ is denoted $\partial f(x)$. In this way, a set valued map $\partial f: E \rightarrow E^{*}$ is obtained.

A monotone set $G$ in $E \times E^{*}$ is a subset of $E \times E^{*}$ for which $\left\langle x-y, x^{*}-y^{*}\right\rangle \geqq 0$ whenever $\left(x, x^{*}\right) \in G$ and $\left(y, y^{*}\right) \in G$. A monotone set is called maximal if it is not properly contained in any other monotone subset of $E \times E^{*}$. By Zorn's lemma any monotone set is contained in a maximal monotone set.

If $f$ is a convex function on $E$, then $g r \partial f=\left\{\left(x, x^{*}\right) \mid x^{*} \in \partial f(x)\right\}$ is a monotone subset of $E \times E^{*}$. Rockafellar $[11,10]$ showed that $g r \partial f$ is actually maximal monotone if $f$ is a l.s.c. proper convex function and $E$ is a Banach space.

If $N$ is a closed subspace of $E$, then $N^{\perp}$ will denote the annihilator of $N$ in $E^{*}$, i.e., $N^{\perp}=\left\{n^{*} \in E^{*} \mid\left\langle n, n^{*}\right\rangle=0\right.$ for each $\left.n \in N\right\}$. If $f$ is a convex function on $E$, then the conjugate of $f$ is the function $f^{*}: E^{*} \rightarrow R \cup\{\infty\}$ defined by $f^{*}\left(x^{*}\right)=\sup \left\{\left\langle x, x^{*}\right\rangle-f(x) \mid x \in E\right\}$.

We recall that the following three statements are equivalent [9]: $z^{*} \in \partial f(z), z \in \partial f^{*}\left(z^{*}\right)$, and $f^{*}\left(z^{*}\right)+f(z)=\left\langle z, z^{*}\right\rangle$.

If $C \subset E$, the indicator function $\psi_{C}$ for $C$ is defined for each $x \in E$ by $\psi_{C}(x)=0$ if $x \in C$ and by $\psi_{C}(x)=\infty$ if $x \notin C$.

If $C \subset E^{*}$, the support function $S_{C}$ for $C$ is defined for each $x \in E$ by $S_{C}(x)=\sup \left\{\left\langle x, x^{*}\right\rangle \mid x^{*} \in C\right\}$.

If $C$ is a nonempty convex subset of $E$, then $0^{+} C$ will denote the asymptotic cone of $C$, i.e., $0^{+} C=\{y \in E \mid x+\lambda y \in C$ for each $\lambda \geqq 0$ and $x \in C\}$.

The following lemma of Phelps [7] is a geometric formulation of the lemma of Brøndsted-Rockafellar [2].

Lemma 1.1. Let $C$ be a closed convex subset of the Banach space E. Suppose $x \in C, x^{*} \in E^{*}$ and $\varepsilon>0$ satisfy

$$
\sup x^{*}(C) \leqq\left\langle x, x^{*}\right\rangle+\varepsilon \text {. }
$$

Then for any $k>0$ there exist $w \in C$ and $w^{*} \in E^{*}$ satisfying

$$
\left\langle w, w^{*}\right\rangle=\sup w^{*}(C),\|x-w\| \leqq \varepsilon / k \text { and }\left\|x^{*}-w^{*}\right\| \leqq k .
$$

We remark that Lemma 1 of [12] and the result [1, Theorem 2] which inspired it are easy consequences of the above lemma.

We show next that the lemma yields a short proof of [1, Theorem 2].

Theorem 1.2. [Bishop-Phelps]. Suppose that $C$ and $X$ are subsets of a Banach space $E$, that $C$ is closed and convex and that $X$ is bounded and nonempty. If $\varepsilon>0$ and if $x^{*} \in E^{*}$ is such that 


$$
\sup x^{*}(C)<\inf x^{*}(X)
$$

then there exist $w^{*} \in E^{*}$ and $w \in C$ such that

$$
\left\|x^{*}-w^{*}\right\| \leqq \varepsilon \text { and }\left\langle w, w^{*}\right\rangle=\sup w^{*}(C)<\inf w^{*}(X) .
$$

Proof. Let $\delta=\inf x^{*}(X)-\sup x^{*}(C)$; then $\delta>0$ and we can assume $2 \varepsilon-\delta<0$. Choose $z \in C$ such that $\sup x^{*}(C) \leqq\left\langle z, x^{*}\right\rangle+\varepsilon$. Since $X$ is bounded, there is a number $N$ such that

$$
N-1>\sup \{\|z-x\| \mid x \in X\} \text {. }
$$

Let $k=\varepsilon / N$ in Lemma 1.1 ; then there exist $w^{*} \in E^{*}$ and $w \in C$ such that

$$
\sup w^{*}(C)=\left\langle w, w^{*}\right\rangle,\left\|w^{*}-x^{*}\right\| \leqq \varepsilon / N \text { and }\|w-z\| \leqq N .
$$

Since $N>1$, we have $\varepsilon / N<\varepsilon$, so it only remains to show that

$$
\left\langle w, w^{*}\right\rangle=\sup w^{*}(C)<\inf w^{*}(X) .
$$

First notice that

$$
\left\langle x, x^{*}\right\rangle-\left\langle z, x^{*}\right\rangle \leqq(\varepsilon / N)\|x-z\|+\left\langle x, w^{*}\right\rangle-\left\langle z, w^{*}\right\rangle
$$

for any $x \in X$ (since $\left.\left\|w^{*}-x^{*}\right\| \leqq \varepsilon / N\right)$. This implies that

$$
\left\langle z, w^{*}\right\rangle-\left\langle z, x^{*}\right\rangle+\inf x^{*}(X) \leqq \varepsilon+\inf w^{*}(X) .
$$

Similarly, $\sup w^{*}(C)=\left\langle w, w^{*}\right\rangle \leqq\left\langle z, w^{*}\right\rangle+(\varepsilon / N)\|w-x\|-\left\langle z, x^{*}\right\rangle+$ $\left\langle w, x^{*}\right\rangle$ which combines with (1) to yield

$$
\begin{aligned}
\left\langle w, w^{*}\right\rangle & \leqq \varepsilon+\inf w^{*}(X)-\inf x^{*}(X)+\varepsilon+\sup x^{*}(C) \\
& \leqq 2 \varepsilon-\delta+\inf w^{*}(X) \\
& <\inf w^{*}(X) .
\end{aligned}
$$

Proposition 1.3 (below) yields a slightly generalized version of Taylor's theorem [12]. The proof of the proposition, although technical, is conceptually very simple: one separates epi $f$ from an appropriate subset of a given linear variety and then uses Lemma 1.1 to obtain a supporting hyperplane of the desired type. In order to facilitate this idea, we introduce some notation.

Suppose that $N$ is a closed subspace of the Banach space $E$, that $B_{N}$ is the unit ball of $N$, and that $f$ is a convex function on $E$. For each $\varepsilon>0$, we let

$$
S(f, \varepsilon, N)=\left\{z^{*} \in E^{*} \mid \sup \left(z^{*},-1\right)(\operatorname{epi} f) \leqq \inf \left(z^{*},-1\right)\left(B_{N} \times\{-\varepsilon\}\right)\right\} \text {. }
$$

Thus, $S(f, \varepsilon, N)$ is the projection onto $E^{*}$ of those functionals in $E^{*} \times\{-1\}$ which separate the convex set epi $f$ from the convex set 
$B_{N} \times\{-\varepsilon\}$. It can also be described by $\left\{z^{*} \in E^{*} \mid f^{*}\left(z^{*}\right)+\left\|z^{*}\right\|_{N} \leqq \varepsilon\right\} \quad$ where $\left\|z^{*}\right\|_{N}=\sup \left\{\mid\left\langle n, z^{*}\right\rangle \| n \in B_{N}\right\}$.

Proposition 1.3. Let $f$ be a l.s.c. proper convex function on a Banach space $E$ and let $N$ be a closed subspace of $E$. Suppose that $f(0)=0$ and that $z^{*} \in S(f, \varepsilon, N)$. Then for each $k \in(0,1)$, there exist $w \in E$ and $w^{*} \in E^{*}$ such that

$$
\begin{aligned}
& w^{*} \in \partial f(w),\left|\left\langle w, w^{*}\right\rangle\right| \leqq(\varepsilon / k)\left(\frac{1+k}{1-k}\right),\left\|w^{*}\right\|_{N} \leqq \frac{k+\varepsilon}{1-k} \\
& \|w\| \leqq \varepsilon / k,|f(w)| \leqq \varepsilon / k,\left\|z^{*}-w^{*}\right\| \leqq \frac{k\left(1+\left\|z^{*}\right\|\right)}{1-k} .
\end{aligned}
$$

Proof. Since $z^{*} \in S(f, \varepsilon, N)$ and $(0,0) \in$ epi $f$, it follows that, $0 \leqq \sup \left(z^{*},-1\right)($ epi $f) \leqq-\left\|z^{*}\right\|_{N}+\varepsilon$ and hence that $\sup \left(z^{*},-1\right)$ (epi $f$ ) $\leqq \varepsilon$ and $\left\|z^{*}\right\|_{N} \leqq \varepsilon$. By Lemma 1.1, for any $k>0$ there exist $G \in(E \times R)^{*}$ and $(w, f(w)) \in$ epi $f$ such that

$$
\sup G(\operatorname{epi} f)=G(w, f(w)),\|(w, f(w))\| \leqq \varepsilon / k \text { and }\left\|\left(z^{*},-1\right)-G\right\| \leqq k \text {. }
$$

Thus $|G(0,-1)-1| \leqq k$ and since $k<1$ we have $0<1-k<G(0,-1)$. Let $\alpha=(G(0,-1))^{-1}$; then there is an element $w^{*} \in E^{*}$ such that for each $y \in E$

$$
\left\langle y, w^{*}\right\rangle=\alpha G(y, 0)
$$

If $y \in \operatorname{dom} f$, then

$$
\begin{aligned}
\left\langle y, w^{*}\right\rangle-f(y) & =\alpha G(y, 0)-f(y) \\
& =\alpha G(y, f(y)) \\
& \leqq \alpha G(w, f(w)) \\
& =\left\langle w, w^{*}\right\rangle-f(w)
\end{aligned}
$$

and therefore $w^{*} \in \partial f(w)$.

Since $(w, f(w)) \in \operatorname{epi} f$, we have $\left\langle w, z^{*}\right\rangle-f(w) \leqq \varepsilon$; thus

$$
\begin{aligned}
0 \leqq G(w, f(w)) & \leqq\|(w, f(w))\| k+\left\langle w, z^{*}\right\rangle-f(w) \\
& \leqq \varepsilon+\varepsilon=2 \varepsilon
\end{aligned}
$$

and therefore

$$
\begin{aligned}
\left|\left\langle w, w^{*}\right\rangle\right| & =|\alpha G(w, 0)|=|\alpha G(w, f(w))+f(w)| \\
& \leqq \frac{2 \varepsilon}{1-k}+\frac{\varepsilon}{k}=\frac{\varepsilon}{k}\left(\frac{1+k}{1-k}\right) .
\end{aligned}
$$

Because $\left\|z^{*}-G(0,-1) w^{*}\right\| \leqq k$, the triangle inequality yields 


$$
\begin{aligned}
\left\|z^{*}-w^{*}\right\| & \leqq k+(1-G(0,-1))\left\|w^{*}\right\| \\
& \leqq k+k \alpha\left(k+\left\|z^{*}\right\|\right) \\
& \leqq k\left(1+\frac{k+\left\|z^{*}\right\|}{1-k}\right) \\
& =\frac{k}{1-k}\left(1+\left\|z^{*}\right\|\right) .
\end{aligned}
$$

Clearly,

$$
\left\|w^{*}\right\|_{N}=\alpha\|G\|_{N \times\{0\}} \leqq \frac{k+\left\|z^{*}\right\|_{N}}{1-k} \leqq \frac{k+\varepsilon}{1-k}
$$

and the proof is complete.

Corollary 1.4. Suppose that $f$ is a l.s.c. proper convex function on a Banach space $E$, that $f(0)=0$, and that $N$ is a closed subspace. Then the following two statements are equivalent:

(1) For each $\varepsilon>0$ the set $S(f, \varepsilon, N)$ is nonempty.

(2) For each $\varepsilon>0$ there exist $w \in E$ and $w^{*} \in E^{*}$ such that $w^{*} \in \partial f(w),\left|\left\langle w, w^{*}\right\rangle\right| \leqq \varepsilon,\|w\| \leqq \varepsilon,\left\|w^{*}\right\|_{N} \leqq \varepsilon$ and $|f(w)| \leqq \varepsilon$.

Proof. (1) $\rightarrow$ (2). We can suppose that $0<\varepsilon<1$. Choose $\delta>0$ so that $\left(\delta^{1 / 2}+\delta\right) /\left(1-\delta^{1 / 2}\right)<\varepsilon$. Let $x^{*} \in S(f, \delta, N)$ and apply Proposition 1.3 with $k=\delta^{1 / 2}$. Since $\delta^{1 / 2}<\left(\delta^{1 / 2}+\delta\right) /(1-\delta)<\varepsilon$ assertion (2) follows.

$(2) \rightarrow(1)$. Let $\varepsilon>0$ and apply (2) with $\varepsilon / 3$. Since $w^{*} \in \partial f(w)$ we have $f^{*}\left(w^{*}\right)=\left\langle w, w^{*}\right\rangle-f(w)$, so that

$$
\begin{aligned}
f^{*}\left(w^{*}\right)+\left\|w^{*}\right\|_{N} & \leqq\left\langle w, w^{*}\right\rangle-f(w)+\left\|w^{*}\right\|_{N} \\
& \leqq \varepsilon / 3+\varepsilon / 3+\varepsilon / 3=\varepsilon
\end{aligned}
$$

and therefore $w^{*} \in S(f, \varepsilon, N)$.

It is now easy to prove the more general version of the theorem of Taylor [12] referred to earlier. It is readily seen that (1) of Corollary 1.4 is equivalent to (1)' For each $\varepsilon>0$ we have

$$
0 \notin \operatorname{cl}\left(B_{N} \times\{-\varepsilon\}-\operatorname{epi} f\right),
$$

which is in turn equivalent to saying that there exists a hyperplane in $E \times R$ which strictly separates epi $f$ from $B_{N} \times\{-\varepsilon\}$. This suggests that a generalization of Taylor's theorem is not possible for arbitrary closed subspaces $N$ and the example following Theorem 1.5 shows that this is indeed the case. In [12], the space $N$ is assumed to be one dimensional. 
THEOREM 1.5. Suppose $f$ is a l.s.c. proper convex function on a Banach space $E$ and that $N$ is a reflexive subspace of $E$. Suppose also that $y \in \operatorname{dom} f$ and $y^{*} \in E^{*}$ are such that for each $n \in N$

$$
f(y+n) \geqq f(y)+\left\langle n, y^{*}\right\rangle \text {. }
$$

Then for each $\varepsilon>0$ there exist $z \in E$ and $z^{*} \in E^{*}$ satisfying

$$
z^{*} \in \partial f(z),\left\|z^{*}-y^{*}\right\|_{N} \leqq \varepsilon,\|y-z\| \leqq \varepsilon \text { and }\left|\left\langle y-z, z^{*}\right\rangle\right| \leqq \varepsilon .
$$

Proof. Let $h=f(y+\cdot)-f(y)-\langle\cdot, y\rangle$; then $h$ is a l.s.c. proper convex function on $E, h(0)=0$ and $h \geqq 0$ on $N$. Hence the weakly closed convex set epi $h$ is disjoint from the weakly compact convex set $B_{N} \times\{-\varepsilon\}$ for every $\varepsilon>0$. By the separation theorem, there is a $G \in(E \times R)^{*}$ such that

$$
\sup G(\operatorname{epi} h)<\inf G\left(B_{N} \times\{-\varepsilon\}\right) .
$$

Since $(0,0) \in$ epi $h$, we have $G(0,-1)>0$. Thus for any $\varepsilon>0$, the set $S(h, \varepsilon, N)$ is nonempty. By applying Corollary 1.4, with $\delta=$ $\varepsilon /\left(1+\left\|y^{*}\right\|\right)$ in place of $\varepsilon$, we obtain $w \in E$ and $w^{*} \in E^{*}$ such that

$$
w^{*} \in \partial h(w),\left|\left\langle w, w^{*}\right\rangle\right| \leqq \delta,\|w\| \leqq \delta \text { and }\left\|w^{*}\right\|_{N} \leqq \delta .
$$

Let $z^{*}=w^{*}+y^{*}$ and $z=w+y$; it is easy to check that $z^{*}$ and $z$ satisfy the conclusion of the theorem.

We refer the reader to [12] for the application of this result to an easy proof of Rockafellar's theorem that for any l.s.c. proper convex function $f$ on a Banach space, the subdifferential map $\partial f$ is maximal monotone.

The following is an example of a l.s.c. proper convex function $F$ and a subspace $N$ of codimension 1 for which $F(0)=0$ and $F(n) \geqq 0$ for all $n \in N$, but (1) of Corollary 1.4 does not hold. This shows that Theorem 1.5 is not valid for arbitrary closed subspaces $N$.

Let

$$
E=l^{\infty} \quad \text { and } \quad N=\left\{x=\left\{x_{i}\right\} \in l^{\infty} \mid x_{1}=0\right\} .
$$

Define $F: l^{\infty} \rightarrow R \cup\{\infty\}$ by

$$
F(x)=\lim _{\varepsilon \rightarrow 0} \inf \{f(y) \mid\|y-x\|<\varepsilon\}
$$

where, for $y=\left\{y_{i}\right\} \in l^{\infty}$,

$$
f(y)=-\left(\prod_{i=1}^{n}\left\{y_{i}\right\}\right)^{1 / n} \quad \text { if } \quad y_{i} \in[0,1]
$$


for each $i$ and $y_{i}=0$ for almost all $i$ ( $n$ is the smallest positive integer such that $i>n$ implies $\left.y_{i}=0\right) ; f(y)=\infty$ otherwise. We have using [11, p. 27] that $f$ is convex and hence $F$ is a l.s.c. proper convex function. Also, $F(0)=0$ and $F(n) \geqq 0$ for each $n \in N$. Let

$$
y_{k}=\{\overbrace{\varepsilon^{k}, \cdots, \varepsilon^{k}}^{k}, \frac{k^{2}-k}{1,1,1, \cdots 1}, 0 \cdots
$$

and $m_{k}=y_{k}-\varepsilon^{k} e_{1}$ where $e_{1}=\{1,0 \cdots\}$. Then $F\left(y_{k}\right)=-\varepsilon$ for each $k$, and $m_{k}-y_{k} \rightarrow 0$. Thus $0 \in \operatorname{cl}\left(B_{N} \times\{-\varepsilon\}-\right.$ epi $\left.F\right)$ and the discussion preceding Theorem 1.5 shows that the conclusion of Theorem 1.5 does not hold.

We remark that if $f$ is continuous at some point of $N$, then as has been noted by Ioffe [5, Theorem 1] $\left.\partial f\right|_{N}=\gamma \circ \partial f$ on $N$ where $\gamma: E^{*} \rightarrow E^{*} / N^{\perp}$ is the canonical projection. Our example shows that continuity cannot be weakened to lower semicontinuity even if one supposes $N$ has codimension one, where [1, Lemma 4] applies. Since $\left.0 \in \partial F\right|_{N}(0)$, we have to show that if $y^{*} \in \partial F(0)$, then $y^{*} \notin N^{\perp}$. Suppose $y^{*} \in \partial F(0)$ i.e., $\left\langle x, y^{*}\right\rangle \leqq F(x)$ for each $x \in E$. Take $k$ large enough so that $\left|\left\langle m_{k}-y_{k}, y^{*}\right\rangle\right|<\varepsilon / 2$; then for large enough $k$,

$$
\begin{aligned}
\left\langle m_{k}, y^{*}\right\rangle & <\varepsilon / 2+\left\langle y_{k}, y^{*}\right\rangle \\
& \leqq \varepsilon / 2+F\left(y_{k}\right) \\
& =-\varepsilon / 2
\end{aligned}
$$

and so $y^{*} \notin N^{\perp}$.

We now give a sufficient condition for $w^{*}$ in Corollary 1.4 (2) to be contained in $N^{-}$i.e. $\left\|w^{*}\right\|_{N}=0$. This result (Proposition 1.8) follows (in the same way Proposition 1.2 was a consequence of Lemma 1.1) from a lemma which extends to Lemma 1.1. We need the following proposition of Dieudonné [3] for the proof of the lemma.

Proposition 1.6. Let $E$ be a topological vector space and $A, B$ two closed convex and nonempty subsets of $E$. Suppose $A$ is locally compact and that $0^{+} A \cap 0^{+} B=\{0\}$. Then $B-A$ is closed in $E$.

Lemma 1.7. Let $C$ be a closed convex subset of a Banach space $E$ and $N$ a finite dimensional subspace of $E$. Suppose $0^{+} C \cap N=\{0\}$ and that $x^{*} \in N^{\perp}, x \in C$ and $\varepsilon>0$ are such that

$$
\sup x^{*}(C) \leqq\left\langle x, x^{*}\right\rangle+\varepsilon \text {. }
$$

Then for any $k>0$, there exist $w \in C$ and $w^{*} \in N^{\perp}$ such that

$$
\left\langle w, w^{*}\right\rangle=\sup w^{*}(C),\|x-w\|_{N^{\perp}} \leqq \varepsilon / k \quad \text { and } \quad\left\|w^{*}-x^{*}\right\| \leqq k .
$$


Proof. By Proposition 1.6, the set $C+N$ is closed in $E$. Let $Q: E \rightarrow E / N$ be the quotient map. Since $Q^{-1}(Q(C))=C+N$, it follows from the definition of the quotient topology that $Q(C)$ is closed. We identify $(E / N)^{*}$ with $N^{\perp}$ and apply Lemma 1.1 to $Q(C)$ in $E / N$. Then for any $k>0$ there exist $z+N \in C+N$ and $w^{*} \in N^{\perp}$ such that

$$
\left\langle z+N, w^{*}\right\rangle=\sup w^{*}(C+N),\|x-z+N\| \leqq \varepsilon / k \text { and }\left\|z^{*}-x^{*}\right\| \leqq k \text {. }
$$

Since $z+N \in C+N$, there exist $w \in C$ and $n \in N$ for which $z=w+n$. Because $w^{*} \in N^{\perp}$, we have

$$
\left\langle w, w^{*}\right\rangle=\left\langle z+N, w^{*}\right\rangle=\sup w^{*}(C+N)=\sup w^{*}(C) .
$$

Also, we have

$$
\|x-w\|_{N^{\perp}}=\|x-w+N\|=\|x-z+N\| \leqq \varepsilon / k,
$$

and the proof is complete.

Proposition 1.8. Let $f$ be a l.s.c. proper convex function on a Banach space $E$ and let $N$ be a finite dimensional subspace of $E$. Suppose that $z^{*} \in S(f, \varepsilon, N)$, that $0^{+}$epi $f \cap(N \times\{0\})=\{0\}$, that $(0,0) \in$ epi $f$ and that $z^{*} \in N^{\perp}$. Then for each $k \in(0,1)$ there exist $w \in E$ and $w^{*} \in N^{\perp}$ such that

$$
w^{*} \in \partial f(w),\|w\|_{N^{\perp}} \leqq \varepsilon / k,|f(w)| \leqq \varepsilon / k
$$

and

$$
\left\|z^{*}-w^{*}\right\| \leqq \frac{k\left(1+\left\|z^{*}\right\|\right)}{1-k}
$$

Proof. By hypothesis, $\sup \left(z^{*},-1\right)($ epi $f) \leqq \varepsilon$ and $\left(z^{*},-1\right) \in$ $(N \times\{0\})^{\perp}=N^{\perp} \times R$ and Lemma 1.7 applies with $x=(0,0)$. Thus for any $k>0$ there exist $(w, f(w)) \in \operatorname{epi} f$ and $G \in N^{\perp} \times R$ such that

and

$$
G(w, f(w))=\sup G(C),\|(w, f(w))\|_{N^{\perp} \times R} \leqq \varepsilon / k
$$

$$
\left\|G-\left(z^{*},-1\right)\right\| \leqq k
$$

Thus $|G(0,-1)-1| \leqq k$ and since $k<1$ we have $0<1-k<G(0,-1)$. Hence there is an element $w^{*} \in E^{*}$ such that for each $y \in E$

$$
\left\langle y, w^{*}\right\rangle=\frac{G(y, 0)}{G(0,-1)} .
$$

Since $G \in N^{\perp} \times R$, we have $w^{*} \in N^{\perp}$; the verifications that $w^{*} \in \partial f(w)$ and that 


$$
\left\|z^{*}-w^{*}\right\| \leqq \frac{k\left(1+\left\|z^{*}\right\|\right)}{1-k}
$$

are the same as in Proposition 1.3.

It is a trivial consequence of Lemma 1.1 that if $C$ is a closed convex subset of a Banach space $E$, then the support functionals of $C$ are norm dense in the set of linear functionals bounded above on $C$. The following corollary to Proposition 1.8 shows that the support functionals of $C$ which are bounded above on $C$ and positive at some point of $C$ have a norm dense intersection with any finite codimensional linear variety $M$ satisfying $0^{+} C \cap 0^{+} M^{0}=\{0\}$.

Corollary 1.9. Let $C$ be a closed convex and nonempty subset of a Banach space $E$ and let $N$ be a finite dimensional subspace of $E$ with $0^{+} C \cap N=\{0\}$. Let $x^{*} \in E^{*}$ be such that $0<S_{C}\left(x^{*}\right)<\infty$. Then for each $\varepsilon>0$ there exist $z^{*} \in E^{*}$ and $z \in C$ satisfying

and

$$
\left\langle z, z^{*}\right\rangle=\sup z^{*}(C), \quad z^{*} \in N^{\perp}+x^{*}, \quad\left\|z^{*}-x^{*}\right\| \leqq \varepsilon
$$

$$
\left|S_{C}\left(x^{*}\right)-S_{C}\left(z^{*}\right)\right| \leqq \varepsilon
$$

Proof. Choose $\delta \in\left(0, S_{C}\left(x^{*}\right)\right)$ such that $2 \delta^{1 / 2} /\left(1-2 \delta^{1 / 2}\right)<\varepsilon$ and $y \in C$ so that

$$
S_{C}\left(x^{*}\right) \leqq\left\langle y, x^{*}\right\rangle+\delta
$$

Define the function $h: E \rightarrow R \cup\{\infty\}$ by $h(x)=\psi_{C}(x)-\left\langle x, x^{*}\right\rangle+\left\langle y, x^{*}\right\rangle$. Clearly (1) implies $0 \in S(h, \delta, N)$. We check that

$$
0^{+} \text {epi } h \cap((N \times R\{y\}) \times\{0\})=\{0\} .
$$

Suppose $(n, 0) \in 0^{+}$epi $h$ for $n \in N$; then since $h(y)=0$, we have for any $\lambda \geqq 0$ that $(y+\lambda n, 0) \in$ epi $h$ i.e., $h(y+\lambda n) \leqq 0$. Hence $\psi_{c}(y+\lambda n) \leqq$ $\left\langle\lambda n, x^{*}\right\rangle<\infty$ and therefore $y+\lambda n \in C$. Thus $n \in 0^{+} C \cap N=\{0\}$. Suppose $(y, 0) \in 0^{+}$epi $h$; then we have $((1+\lambda) y, 0) \in$ epi $h$ for each $\lambda \geqq 0$ and hence $y+\lambda y \in C$ for each $\lambda \geqq 0$; but since $\left\langle y, x^{*}\right\rangle>0$ this contradicts $S_{C}\left(x^{*}\right)<\infty$. Finally suppose $(-y, 0) \in 0^{+}$epi $h$; then $((1-\lambda) y, 0) \in \operatorname{epi} h$ for each $\lambda \geqq 0$, so $\left\langle\lambda y, x^{*}\right\rangle \leqq 0$ for each $\lambda \geqq 0$ which contradicts $\left\langle y, x^{*}\right\rangle>0$.

We can apply Proposition 1.8 with $k=2 \delta^{1 / 2}$ to obtain $w^{*} \in(N x R\{y\})^{\perp}$ and $z \in C$ satisfying $w^{*} \in \partial h(z)$ and $\left\|w^{*}\right\| \leqq 2 \delta^{1 / 2} /\left(1-2 \delta^{1 / 2}\right)<\varepsilon$ and $|h(z)| \leqq \delta^{1 / 2} / 2$ and $\|z\|_{(N \times R\{y\})^{\perp}} \leqq \delta / 2$. Let $z^{*}=w^{*}+x^{*}$; then $\left\|z^{*}-x^{*}\right\| \leqq \varepsilon$ and it is easy to check that $w^{*} \in \partial h(z)$ implies $\left\langle z, z^{*}\right\rangle=S_{C}\left(z^{*}\right)$. Since $h(z)=\left\langle y-z, x^{*}\right\rangle$ we have 


$$
\begin{aligned}
0 & \leqq S_{C}\left(z^{*}\right)-\left\langle y, z^{*}\right\rangle \\
& =S_{C}\left(z^{*}\right)-\left\langle y, x^{*}\right\rangle \\
& =\left\langle z-y, z^{*}\right\rangle \\
& =\left\langle z, w^{*}\right\rangle+\left\langle z-y, x^{*}\right\rangle \\
& \leqq \delta^{1 / 2}<\varepsilon,
\end{aligned}
$$

and combining this inequality with

$$
-\delta \leqq\left\langle y, z^{*}\right\rangle-S_{C}\left(x^{*}\right) \leqq 0
$$

we obtain

$$
\left|S_{C}\left(z^{*}\right)-S_{C}\left(x^{*}\right)\right| \leqq \varepsilon
$$

which completes the proof.

2. In this section we obtain dual results for most of those in $\S 1$. The key is the following lemma which is a dual version of Lemma 1.1. It was essentially proved in [8, Theorem 1] and can be easily obtained from the Brondsted-Rockafellar lemma.

Lemma 2.1. Let $C$ be a weak*closed convex subset of the dual $E^{*}$ of the Banach space $E$. Suppose $x^{*} \in C$ and $x \in E$ and $\varepsilon>0$ satisfy

$$
\sup C(x) \leqq\left\langle x, x^{*}\right\rangle+\varepsilon .
$$

Then for any $k>0$ there exist $w^{*} \in C$ and $w \in E$ satisfying

$$
\left\langle w, w^{*}\right\rangle=\sup C(w),\|x-w\| \leqq k \text { and }\left\|x^{*}-w^{*}\right\| \leqq \varepsilon / k .
$$

This lemma can be used to prove the following dual version of Proposition 1.3, by much the same method.

Proposition 2.2. Let $f$ be a l.s.c. proper convex function on a Banach space $E$ and $N$ a subspace of $E$. Suppose $f^{*}(0)=0$ and $z \in S\left(f^{*}, \varepsilon, N^{\perp}\right)$ where $\varepsilon>0$. Then for each $k \in(0,1)$ there exist $w \in E$ and $w^{*} \in E^{*}$ satisfying:

$$
\begin{aligned}
w & \in \partial f^{*}\left(w^{*}\right) \\
\left|\left\langle w, w^{*}\right\rangle\right| & \leqq(\varepsilon / k)\left(\frac{1+k}{1-k}\right) \\
\|w\|_{N^{\perp}} & \leqq \frac{k+\varepsilon}{1-k} \\
\left\|w^{*}\right\| & \leqq \varepsilon / k \\
\|z-w\| & \leqq \frac{k(1+\|z\|)}{1-k} .
\end{aligned}
$$


Proof. Since $z \in S\left(f^{*}, \varepsilon, N^{\perp}\right)$ and $(0,0) \in$ epi $f^{*}$, it follows that $0 \leqq \sup (z,-1)\left(\right.$ epi $\left.f^{*}\right) \leqq-\|z\|_{N^{\perp}}+\varepsilon$ and hence that

$$
\sup (z,-1)\left(\text { epi } f^{*}\right) \leqq \varepsilon \text { and }\|z\|_{N^{\perp}} \leqq \varepsilon .
$$

By Lemma 2.1, for any $k>0$ there exist $G \in E \times R$ and $\left(w^{*}, f^{*}\left(w^{*}\right)\right) \in$ epi $f^{*}$ satisfying

$$
\sup G\left(\operatorname{epi} f^{*}\right)=G\left(w^{*}, f^{*}\left(w^{*}\right)\right),\left\|\left(w^{*}, f^{*}\left(w^{*}\right)\right)\right\| \leqq \varepsilon / k
$$

and

$$
\|(z,-1)-G\| \leqq k .
$$

Thus $|G(0,-1)-1| \leqq k$ and since $k<1$, we have $0<1-k<G(0,-1)$. Hence there is an element $w \in E$ such that for each $y^{*} \in E^{*}$

$$
\left\langle w, y^{*}\right\rangle=\frac{G\left(y^{*}, 0\right)}{G(0,-1)} .
$$

The rest of the proof is obtained by interchanging the roles of $w$, and $w^{*}$ in the proof of Proposition 1.3.

We have seen that Theorem 1.5 requires some restriction in the subspace $N$. This is not the case with the following dual version which uses the weak* compactness of the unit ball of the weak* closed subspace $N^{\perp}$.

Theorem 2.3. Suppose $f$ is a l.s.c. proper convex function on a Banach space $E$ and that $N$ is a subspace of $E$. Suppose also that $y \in E$ and $y^{*} \in \operatorname{dom} f^{*}$ satisfy

$$
f^{*}\left(y^{*}+n^{*}\right) \geqq f^{*}\left(y^{*}\right)+\left\langle y, n^{*}\right\rangle \text { for each } n^{*} \in N^{\perp} .
$$

Then for each $\varepsilon>0$ there exist $z \in E$ and $z^{*} \in E^{*}$ satisfying

$$
z \in \partial f^{*}\left(z^{*}\right),\|z-y\|_{N^{\perp}} \leqq \varepsilon,\left\|y^{*}-z^{*}\right\| \leqq \varepsilon
$$

and

$$
\left|\left\langle z, y^{*}-z^{*}\right\rangle\right| \leqq \varepsilon
$$

Proof. Let $h=f^{*}\left(y^{*}+\cdot\right)-f^{*}\left(y^{*}\right)-\langle y, \cdot\rangle$; then $h$ is $w^{*}-1$. s.c. proper and convex. Also $h(0)=0$ and $h \geqq 0$ on $N^{\perp}$. Hence the weak* closed convex set epi $h$ is disjoint from the weak* compact convex set $B_{N^{\perp}} \times\{-\delta\}$ for every $\delta>0$. By the separation theorem, there exists $G \in E \times R$ satisfying

$$
\sup G(\operatorname{epi} h)<\inf G\left(B_{N^{\perp}} \times\{-\varepsilon\}\right) \text {. }
$$

Since $(0,0) \in$ epi $h$, we have $G(0,-1)>0$. Thus for any $\delta>0$, the set $E \cap S\left(h, \delta, N^{\perp}\right)$ is nonempty. Choose $\delta>0$ so that 


$$
\frac{\delta^{1 / 2}+\delta}{1-\delta^{1 / 2}}<\frac{\varepsilon}{1+|y|}
$$

and apply Proposition 2.2 with $\delta$ in place of $\varepsilon$ and $k=\delta^{1 / 2}$, to obtain $w \in E$ and $w^{*} \in E^{*}$ satisfying

$$
w \in \partial f^{*}\left(w^{*}\right),\left|\left\langle w, w^{*}\right\rangle\right| \leqq \delta,\|w\|_{N^{i}} \leqq \delta \quad \text { and } \quad\left\|w^{*}\right\| \leqq \delta .
$$

Let $z^{*}=w^{*}+y^{*}$ and $z=w+y$; it is easy to check that $z^{*}$ and $z$ satisfy the conclusions of the theorem.

If one considers $E$ as a subspace of $E^{* *}$, then $g r \partial f$ is a subset of $E^{* *} \times E^{*}$, as is $\operatorname{gr}\left(\partial f^{*}\right)^{-1}$ where $\operatorname{gr}\left(\partial f^{*}\right)^{-1}=\left\{\left(x^{* *}, x^{*}\right) \mid x^{* *} \in \partial f^{*}\left(x^{*}\right)\right\}$. Since $x^{*} \in \partial f(x)$ if and only if $x \in \partial f^{*}\left(x^{*}\right)$ if is evident that $g r \partial f \subset$ $\operatorname{gr}\left(\partial f^{*}\right)^{-1}$ and it is natural to ask if there is any other relationship between these two sets. Rockafellar [11] (cf. Gossez [4]) has answered this question: $\overline{g r \partial f}=g r\left(\partial f^{*}\right)^{-1}$, where the closure is taken with respect to the product of the $\mathfrak{A}$ topology on $E^{* *}$ and the norm topology on $E^{*}$. The $\mathfrak{A}$ topology on $E^{* *}$ is the weakest topology on $E^{* *}$ containing the weak** topology and for which the norm on $E^{* *}$ is a continuous function. In general, it is not a vector topology.

We will give a short proof of this result of Rockafellar using Theorem 2.3, after first proving an easy lemma.

LEMma 2.4. Let $f$ be a l.s.c. proper convex function on the Banach space $E$. Suppose $f^{*}(0)=0$ and $x^{* *} \in \partial f^{*}(0)$ and $\delta>0$. Then $x^{* *} \in \partial\left(f+\psi_{B}\right)^{*}(0)$, where $B=B\left(0 ;\left\|x^{* *}\right\|+\delta\right)$.

Proof. Let $K=\operatorname{dom} f$ and let $K_{1}$ be the weak** closure of $K$ in $E^{* *}$. By [6, p. 62], we have $\left(\psi_{K}+\|\cdot\|\right)^{* *}=\psi_{K_{1}}+\|\cdot\|$; hence $\inf \{\|x\| \mid x \in K\}=\inf \left\{\left\|z^{* *}\right\| \mid z^{* *} \in K_{1}\right\}$ and it follows that $K \cap B \neq \varnothing$. By [6, p. 62], $\left(f+\psi_{B}\right)^{* *}=f^{* *}+\psi_{B}{ }^{* *}$; hence

$$
\begin{aligned}
0 & =\inf f^{* *} \quad\left(\text { since } f^{*}(0)=0\right) \\
& =f^{* *}\left(x^{* *}\right) \quad\left(\text { since } x^{* *} \in \partial f^{*}(0)\right) \\
& =\left(f+\psi_{B}\right)^{* *}\left(x^{* *}\right) \\
& =\inf \left(f+\psi_{B}\right)^{* *} ; \text { so } \quad 0 \in\left(f+\psi_{B}\right)^{* *}\left(x^{* *}\right) .
\end{aligned}
$$

THEOREM 2.5. [Rockafellar]. Let $f$ be a l.s.c. proper convex function on the Banach space $E$. Consider $\left(\partial f^{*}\right)^{-1}: E^{* *} \rightarrow E^{*}$. Then $g r\left(\partial f^{*}\right)^{-1}=\overline{g r f}$ where the closure is taken with respect to the product of the i topology on $E^{* *}$ and the norm topology on $E^{*}$

Proof. The bilinear function $\langle\cdot, \cdot\rangle: E^{* *} x E^{*} \rightarrow R$ is continuous for the $\mathfrak{A} \times$ norm topology, hence $\overline{g r \partial f}$ is monotone. Since we already 
know $\operatorname{gr}\left(\partial f^{*}\right)^{-1}$ is maximal monotone, it suffices to show that

$$
g r\left(\partial f^{*}\right)^{-1} \subset \overline{g r \partial f} .
$$

Let $\left(x^{* *}, x^{*}\right) \in g r\left(\partial f^{*}\right)^{-1}$ and suppose we are given $\delta \in(0,1)$ and $\left\{x_{n}^{*}\right\}_{n=1}^{k}$ in the unit sphere of $E^{*}$. By considering $g^{*}(\cdot)=f\left((\cdot)+x^{*}\right)-f^{*}\left(x^{*}\right)$, if necessary, we can assume $x^{*}=0$ and $f^{*}(0)=0$. Thus $0 \in \partial f^{* *}\left(x^{* *}\right)$ and by Lemma 2.4 we have $0 \in \partial\left(f+\psi_{B}\right)^{* *}\left(x^{* *}\right)$, where

$$
B=B\left(0 ;\left\|x^{* *}\right\|+\delta\right) \text {. }
$$

Choose $y \in E$ such that $\left\langle y, x_{n}^{*}\right\rangle=\left\langle x^{* *}, x_{n}^{*}\right\rangle$ for $n=1, \cdots, k$ and apply Theorem 2.3 to $f+\psi_{B}$ with $y^{*}=0$ and $N^{\perp}=\operatorname{span}\left\{x_{n}^{*}\right\}_{n=1}^{k}$ and $\varepsilon=$ $\varepsilon^{\prime} /\left(1+\left\|x^{* *}\right\|\right)$ where $0<\varepsilon^{\prime}<\delta^{2}$. We then obtain $z^{*} \in E^{*}$ and $z \in E$ satisfying $z^{*} \in \partial\left(f+\psi_{B}\right)(z),\|z-y\|_{N^{\perp}} \leqq \varepsilon,\left|\left\langle z, z^{*}\right\rangle\right| \leqq \varepsilon$ and $\left\|z^{*}\right\| \leqq \varepsilon$. Thus, $z^{*}=u^{*}+t^{*}$ where $u^{*} \in \partial f(z)$ and $t^{*} \in \partial \psi_{B}(z)$. Clearly $z \in x^{* *}+$ $\delta\left(\left\{x_{n}^{*}\right\}_{n=1}^{k}\right)^{\circ}$ and $\|z\| \leqq\left\|x^{* *}\right\|+\delta$ and $\left\|u^{*}\right\| \leqq \delta+\left\|t^{*}\right\|$. We will show $\left\|t^{*}\right\| \leqq \delta$ and this will conclude the proof. Suppose $\left\|t^{*}\right\|>\delta$, then because $u^{*} \in \partial f(z)$ and $0 \in \partial f^{* *}\left(x^{* *}\right)$ we have

$$
0 \leqq\left\langle x^{* *}-z,-u^{*}\right\rangle \text {. }
$$

Hence

$$
\begin{aligned}
\|z\|\left\|t^{*}\right\| & =\left\langle z, t^{*}\right\rangle \leqq\left\langle x^{* *}, t^{*}\right\rangle-\left\langle x^{* *}, z^{*}\right\rangle+\left\langle z, z^{*}\right\rangle \\
& \leqq\left\|x^{* *}\right\|\left\|t^{*}\right\|+\left\|x^{* *}\right\|\left\|z^{*}\right\|+\varepsilon \\
& \leqq\left\|x^{* *}\right\|\left\|t^{*}\right\|+\left(\left\|x^{* *}\right\|+1\right) \varepsilon \\
& \leqq\left\|x^{* *}\right\|\left\|t^{*}\right\|+\varepsilon^{\prime} \\
& <\left\|x^{* *}\right\|\left\|t^{*}\right\|+\delta^{2}
\end{aligned}
$$

so $\|z\|<\left\|x^{* *}\right\|+\delta^{2} /\left\|t^{*}\right\|<\left\|x^{* *}\right\|+\delta$ and since $t^{*} \in \partial \psi_{B}(z)$, we have the contradiction $t^{*}=0$.

Finally we prove a dual version of Proposition 1.8. We require a lemma which is a consequence of Proposition 1.6 and Lemma 2.1.

LEMMA 2.6. Let $C$ be a weak* closed convex subset of the dual of a Banach space $E$ and $N$ a closed finite codimensional subspace of E. Suppose $0^{+} C \cap N^{\perp}=\{0\}$, and that $x^{*} \in C, x \in N$ and $\varepsilon>0$ satisfy

$$
\sup C(x) \leqq\left\langle x, x^{*}\right\rangle+\varepsilon \text {. }
$$

Then for any $k>0$ there exist $z \in N$ and $z^{*} \in C$ satisfying

$$
\left\langle z, z^{*}\right\rangle=\sup C(z),\|x-z\| \leqq k,\left\|x^{*}-z^{*}\right\|_{N} \leqq \varepsilon / k .
$$

Proof. By Proposition 1.6, the set $C+N^{\perp}$ is weak* closed in $E^{*}$. Let $Q: E^{*} \rightarrow E^{*} / N^{\perp}$ be the quotient map. Since $Q^{-1}(Q(C))=$ 
$C+N^{\perp}$, it follows from the definition of the quotient topology that $Q(C)$ is weak* closed. We identify $E^{*} / N^{\perp}$ with $N^{*}$ and apply Lemma 2.1 to $Q(C)$ in $E^{*} / N^{\perp}$. Then for any $k>0$ there exist $w^{*}+N^{\perp} \subset$ $C+N^{\perp}$ and $z \in N$ satisfying $\left\langle z, w^{*}+N^{\perp}\right\rangle=\sup C(z),\|z-x\| \leqq k$ and $\left\|x^{*}-w^{*}+N^{\perp}\right\| \leqq \varepsilon / k$. Let $n^{*} \in N^{\perp}$ be such that $w^{*}+n^{*} \in C$ and set $z^{*}=w^{*}+n^{*}$, so that $\left\langle z, z^{*}\right\rangle=\sup C(z)$. Finally, we have

$$
\left\|x^{*}-z^{*}\right\|_{N}=\left\|x^{*}-z^{*}+N^{\perp}\right\|=\left\|x^{*}-w^{*}+N^{\perp}\right\| \leqq \varepsilon / k .
$$

Proposition 2.7. Let $f$ be a l.s.c. proper convex function on a Banach space $E$. Let $N$ be a closed subspace of $E$ of finite codimension. Suppose that $0^{+}$epi $f^{*} \cap\left(N^{\perp} \times\{0\}\right)=\{0\}$, that $(0,0) \in$ epi $f^{*}$ and that $z \in N \cap S\left(f^{*}, \varepsilon, N^{\perp}\right)$, where $\varepsilon>0$. Then for any $k \in(0,1)$ there exist $w \in N$ and $w^{*} \in E^{*}$ satisfying

$$
\begin{aligned}
w \in \partial f^{*}\left(w^{*}\right) & \\
\|z-w\| & \leqq \frac{k(1+\|z\|)}{1-k} \\
\left\|w^{*}\right\|_{N} & \leqq \varepsilon / k \\
\left|f^{*}\left(w^{*}\right)\right| & \leqq \varepsilon / k .
\end{aligned}
$$

Proof. By hypothesis, $\sup (z,-1)\left(\right.$ epi $\left.f^{*}\right) \leqq \varepsilon$ and $(z,-1) \in N \times R$. We can apply Lemma 2.6 with $x=(0,0)$ and obtain, for any $k>0$, $G \in N \times R$ and $\left(w^{*}, f^{*}\left(w^{*}\right)\right) \in \operatorname{epi} f^{*}$ satisfying

$$
G\left(\left(w^{*}, f^{*}\left(w^{*}\right)\right)=\sup G\left(\operatorname{epi} f^{*}\right),\|G-(z,-1)\| \leqq k\right.
$$

and

$$
\left\|\left(w^{*}, f^{*}\left(w^{*}\right)\right)\right\|_{N \times R} \leqq \varepsilon / k .
$$

Thus $|G(0,-1)-1| \leqq k$ and since $k<1$ we have $0<1-k<G(0,-1)$. Hence there exists an element $w \in E$ such that

$$
\left\langle w, y^{*}\right\rangle=\frac{G\left(y^{*}, 0\right)}{G(0,-1)} \text { for each } y^{*} \in E^{*} .
$$

Since $G \in N \times R$, we have $w \in N$.

The verifications that $w \in \partial f^{*}\left(w^{*}\right)$ and that

$$
\|z-w\| \leqq \frac{k(1+\|z\|)}{1-k}
$$

are the same as in Proposition 2.2.

The author would like to thank Professor R. R. Phelps for his help and encouragement. He would also like to thank Professor R. T. Rockafellar for his comments and corrections of an earlier draft of this paper. 


\section{REFERENCES}

1. Errett Bishop and R. R. Phelps, The support functional of a convex set, Proc. Symp. Pure Math., Amer. Math. Soc., 7 (Convexity) (1963), 27-35.

2. A. Brøndsted and R. T. Rockafellar, On the subdifferentiability of convex functions, Proc. Amer. Math. Soc., 16 (1965), 605-611.

3. J. Dieudonné, Sur la séparation des ensembles convexes, Math. Ann., 163 (1966), 1-3.

4. Jean Pierre Gossez, Operateurs Monotone dons les espaces de Banach, Thesis (1969), Univ. Libre de Bruxelles.

5. A. D. Ioffe, Subdifferentials of restrictions of convex functionals, Uspekhi Mat. Nauk, 25 (1970), 181-182.

6. J. J. Moreau, Fonctionelles convexes, mimeographed lecture notes, College de France, 1967.

7. R. R. Phelps, Support cones in Banach spaces and their applications, to appear.

8. - Weak* support points of convex sets in $E^{*}$, Israel J. Math., 2 (1964), $177-182$.

9. R. T. Rockafellar, Convex Analysis, Princeton Univ. Press, Princeton, N. J., (1969). 10. Characterization of the subdifferentials of convex functions, Pacific J. Math., 17 (1966), 497-510.

11. On the maximal monotonicity of subdifferential mappings, Pacific J. Math., 33 (1970), 209-216.

12. P. D. Taylor, The existence of subgradients for convex functions, to appear in Pacific J. Math.

Received May 26, 1972 and in revised form October 18, 1972.

UNIVERSITY OF WASHINGTON 



\section{PACIFIC JOURNAL OF MATHEMATICS}

\section{EDITORS}

D. Gilbarg and J. Milgram

Stanford University

Stanford, California 94305

R. A. Beaumont

University of Washington

Seattle, Washington 98105
J. DUGUNDJI* Department of Mathematics

University of Southern California Los Angeles, California 90007

RICHARD ARENS

University of California

Los Angeles, California 90024

\section{ASSOCIATE EDITORS}

E. F. BeCKenbach

B. H. NeUMaNN

F. WOLF

K. Yoshida

\section{SUPPORTING INSTITUTIONS}

UNIVERSITY OF BRITISH COLUMBIA

UNIVERSITY OF SOUTHERN CALIFORNIA

CALIFORNIA INSTITUTE OF TECHNOLOGY

UNIVERSITY OF CALIFORNIA

MONTANA STATE UNIVERSITY

STANFORD UNIVERSITY

UNIVERSITY OF TOKYO

UNIVERSITY OF NEVADA

UNIVERSITY OF UTAH

NEW MEXICO STATE UNIVERSITY

WASHINGTON STATE UNIVERSITY

OREGON STATE UNIVERSITY

UNIVERSITY OF OREGON

OSAKA UNIVERSITY

UNIVERSITY OF WASHINGTON

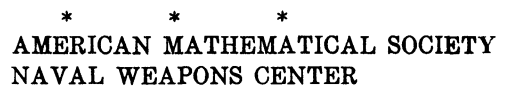

* C. DePrima will replace J. Dugundji until August 1974. 


\section{Pacific Journal of Mathematics}

\section{Vol. 47, No. $1 \quad$ January, 1973}

K. Adachi, Masuo Suzuki and M. Yoshida, Continuation of holomorphic

mappings, with values in a complex Lie group ....................

Michael Aschbacher, A characterization of the unitary and symplectic groups

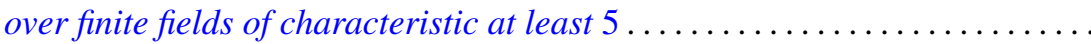

Larry Eugene Bobisud and James Calvert, Energy bounds and virial theorems for abstract wave equations....................................

Christer Borell, A note on an inequality for rearrangements ................

Peter Southcott Bullen and S. N. Mukhopadhyay, Peano derivatives and general

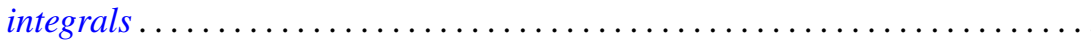

Wendell Dan Curtis, Yu-Lee Lee and Forrest Miller, A class of infinite dimensional subgroups of $\operatorname{Diff}^{r}(X)$ which are Banach Lie groups .........

Paul C. Eklof, The structure of ultraproducts of abelian groups ...............

William Alan Feldman, Axioms of countability and the algebra $C(X) \ldots \ldots \ldots$

Jack Tilden Goodykoontz, Jr., Aposyndetic properties of hyperspaces...........

George Grätzer and J. Płonka, On the number of polynomials of an idempotent algebra. II ...........................................

Alan Trinler Huckleberry, The weak envelope of holomorphy for algebras of

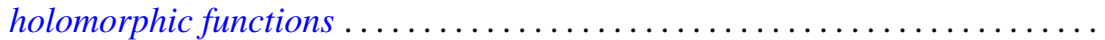

John Joseph Hutchinson and Julius Martin Zelmanowitz, Subdirect sum decompositions of endomorphism rings . . . . . . . . . . . . . . . .

Gary Douglas Jones, An asymptotic property of solutions of

$y^{\prime \prime \prime}+p y^{\prime}+q y=0$.

Howard E. Lacey, On the classification of Lindenstrauss spaces .

Charles Dwight Lahr, Approximate identities for convolution measure algebras.

George William Luna, Subdifferentials of convex functions on Banach

spaces.

Nelson Groh Markley, Locally circular minimal sets. .

Robert Wilmer Miller, Endomorphism rings of finitely generated projective modules

Donald Steven Passman, On the semisimplicity of group rings of linear

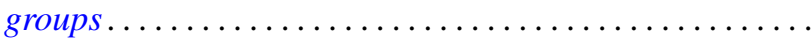

Bennie Jake Pearson, Dendritic compactifications of certain dendritic spaces.

Ryōtarō Satō, Abel-ergodic theorems for subsequences ...... .

Henry S. Sharp, Jr., Locally complete graphs. . .

Harris Samuel Shultz, A very weak topology for the Mikusinski field of

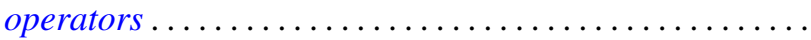

Elena Stroescu, Isometric dilations of contractions on Banach spaces ...

Charles W. Trigg, Versum sequences in the binary system ... . .

William L. Voxman, On the countable union of cellular decompositions of n-manifolds 